\title{
Supernova impostors: LBV outbursts from the most massive stars
}

\author{
Schuyler D. Van Dyk \\ Spitzer Science Center/Caltech, \\ Mail Code 220-6, 1200 E. California Blvd., Pasadena, CA 91125 , USA \\ email: vandyk@ipac.caltech.edu
}

\begin{abstract}
Several recent luminous events that have been identified initially as supernovae $(\mathrm{SNe})$ are probably not genuine $\mathrm{SNe}$ at all. Instead we argue that these events are more likely the outbursts, or super-outbursts, of very massive stars in the luminous blue variable (LBV) phase. At least two of these events are analogous to the Great 1843 Eruption of $\eta$ Carinae.
\end{abstract}

Keywords. supernovae: general, supernovae: individual (SN 1954J/Variable 12, SN 1961V, SN 1997bs, SN 2000ch, SN 2002kg/Variable 37)

The fate of the most massive stars in galaxies is not well known. Such stars have been linked to black hole formation, to some GRBs, and to early metal enrichment. Today, very massive stars are extremely rare, so studying known examples, and finding new ones, in our Galaxy and other nearby galaxies affords us with essential insight into these processes. The Great Eruption of 1843 for $\eta$ Carinae demonstrated that some very massive stars go through spectacular eruptive phases of mass ejection prior to exploding as a SN. For some extragalactic stars observed to date, the energetics of these pre-SN outbursts are comparable to those of SNe themselves, and, hence, these objects have been dubbed SN "impostors". The classical examples are SN 1961V in NGC 1058 and SN 1954J/Variable 12 in NGC 2403. (Zwicky actually considered $\eta$ Car an underluminous SN!) More recent examples include SN 1997bs in NGC 3627 and SN 2000ch in NGC 3432. Although underluminous and photometrically erratic relative to normal, core-collapse $\mathrm{SNe}$, spectroscopically the impostors generally resemble the Type II-narrow SNe, with H emission exhibiting narrow line profiles atop broader bases.

In the case of SN 1954J/V12, using Keck and Hubble Space Telescope data, we have identified the survivor of the super-outburst within a dusty ( $A_{V} \simeq 4 \mathrm{mag}$ ) nebula, analogous to the Homunculus around $\eta$ Car, and have estimated that the star had an initial mass $M_{\text {ini }}>20 \mathrm{M}_{\odot}($ Van Dyk, Li, \& Filippenko 2005, PASP, 117, 553). For SN 1961V it is still unclear whether or not a survivor has been located; a star with a spectrum resembling that of $\eta$ Car has been detected at the SN location, but the presence of nonthermal radio emission, possibly declining in flux, may indicate we are simply seeing an old SN (see Van Dyk 2005, ASP Conf. v. 332, 49, and references therein). For SN 1997bs we actually identified the outburst precursor - however without color information, we can only infer that the highly luminous star $\left(M_{V}=-8.1 \mathrm{mag}\right)$ also had $M_{\mathrm{ini}}>20 \mathrm{M}_{\odot}($ Van Dyk et al. 2000, PASP, 112, 1532). Far less is known about SN 2000ch and any putative outburst survivor. Not all of these outburst events are as extreme as those examples above: SN 2002kg/Variable 37, also in NGC 2403, has properties closer to the more normal, luminous blue variable S Doradus than $\eta$ Car. We have been able to limit the outburst precursor's mass to $M_{\mathrm{ini}}>40 \mathrm{M}_{\odot}$ (Van Dyk et al. 2007, PASP, submitted).

The few known SN impostors so far exhibit a broad range of properties and clearly represent an interesting pre-SN evolutionary phase for very massive stars. 